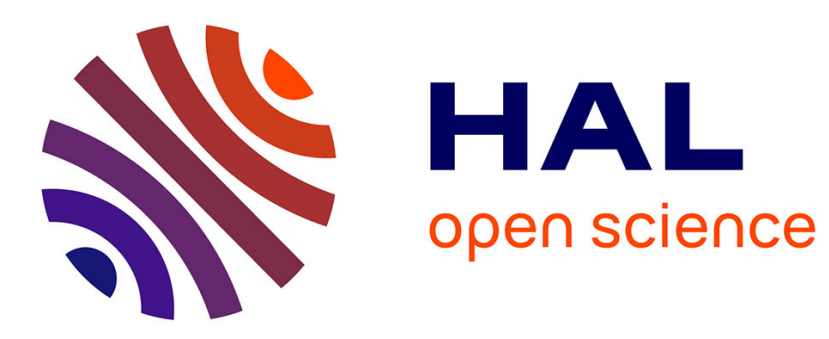

\title{
Eppur si muove, and yet it moves: Patchy (phoretic) swimmers
}

\author{
A. Aubret, S. Ramananarivo, J. Palacci
}

\section{To cite this version:}

A. Aubret, S. Ramananarivo, J. Palacci. Eppur si muove, and yet it moves: Patchy (phoretic) swimmers. Current Opinion in Colloid \& Interface Science, 2017, 30, pp.81 - 89. 10.1016/j.cocis.2017.05.007 . hal-03279186

\section{HAL Id: hal-03279186 \\ https://hal.science/hal-03279186}

Submitted on 6 Jul 2021

HAL is a multi-disciplinary open access archive for the deposit and dissemination of scientific research documents, whether they are published or not. The documents may come from teaching and research institutions in France or abroad, or from public or private research centers.
L'archive ouverte pluridisciplinaire HAL, est destinée au dépôt et à la diffusion de documents scientifiques de niveau recherche, publiés ou non, émanant des établissements d'enseignement et de recherche français ou étrangers, des laboratoires publics ou privés. 


\title{
Eppur si Muove, and Yet it Moves' Patchy (Phoretic) Swimmers
}

\author{
A. Aubret, S. Ramananarivo, J. Palacci ${ }^{2}$ \\ Department of Physics, University of California, San Diego
}

\begin{abstract}
Advances in colloidal synthesis allow for the design of particles with controlled patches. This article reviews routes towards colloidal locomotion, where energy is consumed and converted into motion, and its implementation with active patchy particles. A special emphasis is given to phoretic swimmers, where the self-propulsion originates from an interfacial phenomenon, raising experimental challenges and opening up opportunities for particles with controlled anisotropic surface chemistry and novel behaviors.
\end{abstract}

Keywords: Interfacial Transport, Phoresis, Patchy, Active Colloids.

\section{Introduction}

This review article focuses on active patchy colloids, here defined, as selfpropelling particles a.k.a. swimmers. Self-propulsion comes at a cost: particles need to harvest free energy from their environment and convert it into me5 chanical work. The emergent phenomena, originating from the non-equilibrium nature of the system, are a pivotal issue of the Active Matter field [1, 2] but will not be discussed here. For example, collections of active particles can form clusters [3, 4, "living crystals" [5] or flocks [6] and confined vortices [7]. The advancement of a "thermodynamics" and unified framework for those systems

\footnotetext{
${ }^{1}$ Phrase attributed to Galileo Galilei, commenting on his trial and abjuration. The historic accuracy of the quotation is questionable.

${ }^{2}$ Corresponding Author: palacci@ucsd.edu
}

Preprint submitted to Elsevier

May 22, 2017 
in an ongoing work [8, 9. It notably unveiled a Motility-Induced Phase Separation (MISP), arising from the persistence of the particles, colliding heads-on and driven by the rotational diffusion of the propulsion [10, 11, 12, 13, 14, 15]. Some equilibrium concepts have been extended: active pressure [16, 17, 18, 19, or surface tension [20], but experiments remain unfortunately scarce [21, 22].

15 This article is not intended as an exhaustive review of the swimmers, as available in $223,24,25,26,27$. It is aimed at the colloidal scientist willing to get a better grasp on active colloids, and an insider view on this large body of work. It surveys the different experimental strategies to build self-propelled colloids and focuses on phoretic engines. Our presentation presents a brief description of interfacial transport through a qualitative description of electro-osmosis and broadens the conclusions to the phenomenon of diffusio-phoresis or migration in a solute gradient. We point towards recent results showing that, in practice, model-systems are more complex than originally thought. We conclude by discussing the opportunities offered by patchy phoretic particles.

\section{Self-propelled Colloids}

\subsection{Swimming at the microscale}

Autonomous microswimmers experience low Reynolds number dynamics or "dynamics in a very viscous fluid", following Stokes equation, which is timeindependent [28, 29]. As stated by Purcell in his seminal paper [28, "time doesn't matter, the pattern of motion is the same, whether slow or fast, whether forward or backward in time", and propulsion from a reciprocal stroke, akin to an opening and closing hinge, is precluded ${ }^{3}$. Propulsion can be achieved from non-reciprocal patterns of motion, e.g. wave propagation in an oscillating artificial flagella [30, 31] or corkscrew motion of actuated magnetic structures 35 32, 33, 34, 35. Such systems are macroscopically actuated and the direction of propulsion is set by an external operator. Alternatively, autonomous systems,

\footnotetext{
${ }^{3}$ In a Newtownian fluid
} 
akin to bacteria in Nature, embed a propulsive machinery and propel along a direction set internally. The collective arrangement and the emergence of large scale coherent motion is a key question in non-equilibrium systems and

40 makes artificial swimmers an adequate probe to explore the driving principles of dissipative systems. An effective route toward propulsion at small scale, taking advantage of the miniaturization, is to harness interfacial phenomena. Phoretic effects, by which a field gradient sets colloidal particles into motion, thus appear as a relevant vessel for micro-propulsion. Swimmers propelled by non-interfacial phenomena such as bubbles [36, 37, 38, 39, 40, acoustic waves [41] or ultrasounds [42, 43, or in complex fluids [44, 45, 46, 47, 48, 49, 50, 51] are current topics of research but will not be discussed in this review. An overview of available swimmers is presented on [Fig.1].

\subsection{Individual Dynamics of Autonomous Swimmers}

In the simple case of particles moving in 2D on a substrate, swimmers perform a persistent random walk. The direction of self-propulsion decorrelates over a typical persistence time $\tau_{p}$. Particles exhibit a ballistic motion for $t \ll \tau_{p}$ and an effective diffusive behavior for $t \gg \tau_{p}$ [Fig.2A]. The expression of the $2 \mathrm{D}$ Mean Square Displacement (MSD) from the projection of a 3D motion is given 55 by [52, 53] [Fig.2B]:

$$
\Delta L^{2}(\Delta t)=4 D_{0} \Delta t+\frac{V^{2} \tau_{p}^{2}}{3}\left[\frac{2 \Delta t}{\tau_{p}}+e^{\frac{-2 \Delta t}{\tau_{p}}}-1\right]
$$

where $D_{0}$ is the diffusion coefficient of the particle at equilibrium. The dynamics is analogous to the dynamics of a Brownian particle predicted by a Langevin model, with the persistence time replacing the inertial time scale $(\sim \mathrm{ns})$. Rotational and translational dynamics are often decoupled in the experiment, ${ }_{60} \tau_{p} \sim \tau_{R} \propto R^{3}$, where $\tau_{R}$ is the equilibrium rotational time and $R$, the radius of the particle [53, 54, 5, 55]. The rotational diffusion can be extracted from the MSD, but the uncertainty on the determination from the non-linear fit is often broad. Direct measurement of the rotational dynamics could be alternatively implemented following recent microscopy developments, e.g. confocal 
imaging [56] or differential dynamic microscopy (DDM), originally developed for translational diffusion [57, then bacterial motility [58] and recently adapted to probe rotational dynamics [59].

\section{Basics of Interfacial Transport}

In fluid dynamics, surfaces are often considered as boundary conditions for the fluid domain without taking into account the nanoscopic structure of the layer at the solid/liquid interface. In this section, we introduce physical phenomena demonstrating the impact of this nanometric layer at large scale. The thermodynamic force arising from an interfacial gradient of a field $\mathrm{O}$ can either drive a fluid under motion with respect to a wall by osmosis, or reciprocally move 75 a particle by phoresis. The field $\mathrm{O}$ can be an electric potential $\Phi$, the temperature $\mathrm{T}$, or a solute concentration $\mathrm{c}$, in the cases of respectively, electrophoresis, thermophoresis, and diffusiophoresis.

\subsection{Electro-osmosis for Dummies ${ }^{\circledR}$}

In this section, we discuss the phenomenon of electro-osmosis, i.e. the interfacial flow resulting from the application of an electric field. More detailed derivations and discussions on electrokinetic effects can be found in [60, 61, 62, 63.

A solid surface immersed in an aqueous solution spontaneously gets charged. We consider a negative charge as in the case of silica in water $\left(\mathrm{SiOH}=\mathrm{SiO}^{-}+\mathrm{H}^{+}\right)$. Cations are attracted to the charged surface and anions repelled, leading to the formation of a screening double layer of ions [Fig.3A]. In the Debye-Huckel limit of weak potentials ${ }^{4}$ the electric potential,$\Phi(z)$, decays exponentially from the wall,$\Phi(z)=\zeta e^{-z / \lambda_{D}}$, where $\lambda_{D}^{-2}=2 q^{2} \rho_{0} / \epsilon k_{B} T, \lambda_{D}$ is the Debye length, $\epsilon$ is

\footnotetext{
${ }^{4}$ We assumed a Gouy-Chapman mean field approximation, which neglects the fluctuations of potential, considering only the mean value of the charge distribution, and we neglected the ions-ions and ions-solvent interactions.
} 
the permittivity of the considered medium, $k_{B}$ is the Boltzmann constant, $q$ the charge of the cation and $\rho_{0}$ the bulk concentration of ions 5

The total charge density is approximated by:

$$
\rho= \begin{cases}=0 & \text { if } z \geq \lambda_{d} \\ \neq 0 & \text { if } z<\lambda_{d}\end{cases}
$$

An electric field $E \mathbf{e}_{x}$ is applied along the solid surface [Fig.3A]. The system $S=$ [surface+Debye-Huckel layer+bulk solution] is neutral and so that the total electric force acting on $\mathrm{S}$ is zerd ${ }^{6}, \mathbf{F}_{e}=\int_{S} \rho \mathbf{E d} V=\mathbf{0}$.

The total charge density follows the Poisson-Boltzmann equation, $\Delta \Phi=-\rho / \epsilon$, leading to $\zeta / \lambda_{D}^{2} \sim-\rho / \epsilon$, where the $\zeta$-potential is the potential drop in the Debye layer. The ions in the double layer undergo a net volume force $\mathbf{f}=\rho \mathbf{E}$ and move. The velocity within the layer determines the velocity field outside the layer $\mathbf{V}_{e o}=V_{e o} \mathbf{e}_{x}$. Since $\lambda_{D}$ is nanometric, we rewrite the force as a surface stress: $\sigma_{E} \sim \rho \lambda_{D} \mathbf{E} \sim-\frac{\epsilon \zeta}{\lambda_{D}} \mathbf{E}$. This is the driving power of the motion [Fig.3A]. It is balanced by the viscous stress resulting from the velocity gradient in the double layer, ${ }^{7} \sigma_{\eta} \sim \frac{\eta}{\lambda_{D}} \mathbf{V}_{e o}$, leading to the electro-osmotic velocity:

$$
\mathbf{V}_{e o} \sim-\frac{\epsilon \zeta}{\eta} \mathbf{E}
$$

This result was first derived by Smoluchovski 64].

\subsection{Electro-phoretic motion of a colloidal particle}

We consider the effect of an electric field on a charged colloid of radius $R$. In the thin Debye layer limit $R \gg \lambda_{D}$, the curvature is negligible, and the problem is akin to the electroosmotic flow induced by the tangential electric field, as discussed in the previous section. The particle electrophoretically migrates in the direction opposite to the electro-osmotic flow. Solving the phoretic velocity

\footnotetext{
${ }^{5}$ For a centimolar aqueous solution of a monovalent salt, $\lambda_{D} \sim 3 \mathrm{~nm}$.

${ }^{6}$ Swimming bacteria are an other example of propulsion at zero net force as the drag balances the thrust, and forms, to lowest order, a force dipole.

${ }^{7}$ We consider non-slip boundary conditions at the wall
} 
requires the resolution of the Stokes flow with the appropriate boundary conditions (zero flow at infinity and electro-osmotic slip on the particle surface). It gives, for an homogeneous particle of arbitrary shape, with $\lambda_{D} \ll R$, 65]:

$$
\mathbf{V}_{E P}=\frac{\epsilon \zeta}{\eta} \mathbf{E}=-\mathbf{V}_{E O}
$$

\subsection{Diffusio-osmosis, Diffusio-phoresis}

Neutral Diffusio-osmosis

We extend our understanding of interfacial transport to the phenomenon of diffusio-osmosis: the interfacial flow induced by a concentration gradient of solute. The phenomenon was first reported by Derjaguin in 1947 [66], then by Prieve and coworkers [67, 68. It converts the energy stored in solute gradients into mechanical energy and motion. It is a rather subtle phenomenon, relevant in the variety of natural and artificial situations with concentration gradients, e.g. evaporation, mixing, imposed gradients or asymmetric reactions... In this review, we mainly focus on the results important for the design of active patchy particles, details and examples of diffusio- transport are discussed in [60, 69, 70]. A solid substrate is immersed in a solute solution of bulk concentration $c_{0}$. The solute interacts with the substrate through a potential $U(z)$ with typical range $\lambda$, e.g. $\lambda=\lambda_{D}$, the Debye length, for electrostatics. At thermal equilibrium, the distribution of (non-interacting) solute particles is given by the Boltzmann distribution, $c(z) \approx c_{0} e^{-\frac{U(z)}{k_{B} T}}$. Provided a macroscopic profile $c_{0}(x)$, one can rewrite $c(x, z) \approx c_{0}(x) e^{-\frac{U(z)}{k_{B} T}}$. The resulting unbalanced osmotic pressure in the interaction layer $\lambda$ is the driving force of the diffusio-osmotic flow [FIG.3B]. It is balanced by the viscous stress and leads to the diffusio-osmotic flow [60, 71] :

$$
V_{D O}=-\left(k_{B} T / \eta\right) \Gamma L \frac{d c_{0}}{d x} \mathbf{e}_{\mathbf{x}}=\mu_{D O} \frac{d c_{0}}{d x} \mathbf{e}_{\mathbf{x}}
$$

where $\Gamma=\int_{0}^{\infty} d z\left[e^{-U(z) / k_{B} T}-1\right]$ measures the excess of solute induced by the presence of a solid wall $8 L=\Gamma^{-1} \int_{0}^{\infty} d z z\left[e^{-U(z) / k_{B} T}-1\right]$ is the range of

${ }^{8} \Gamma<0$ for depletion of solute and $\Gamma>0$ for solid-solute attraction 
interaction, and $\mu_{D O}$ the diffusio-osmotic mobility. This description for neutral species is called chemio-osmosis.

In the case of charged species, an additional electrophoretic contribution needs to be accounted for. In an electrolyte gradient, an electric field $\mathbf{E}$ ' is generated to maintain a zero electrical current:

$$
\mathbf{j}_{e}=-(-q) D_{-} \nabla c_{0}-q D_{+} \nabla c_{0}+(-q) c_{0} \mu_{+}^{E} \mathbf{E}^{\prime}+q c_{0} \mu_{-}^{E} \mathbf{E}^{\prime}
$$

with $q>0$ the electrical charge of the cation $9^{9} D_{ \pm}$, the thermal diffusivity of the cations (respectively anions) and $\mu_{ \pm}^{E}$, the electrical mobility of the cations (respectively anions). The fluctuation-dissipation theorem relates the electrical mobility and diffusivity : $\mu_{ \pm}^{E}=\frac{ \pm q D_{ \pm}}{k_{B} T}\left[55\right.$. At zero current, $\mathbf{E}^{\prime}=\beta \frac{k_{B} T}{q} \nabla \log \left(c_{0}\right)$, where the factor $\beta=\frac{D_{+}-D_{-}}{D_{+}+D_{-}}$is ion specific. The charged surface sets an electroosmotic flow in the self-induced electric field $\mathbf{E}$ ':

$$
\mathbf{V}_{E}^{\prime}=-\frac{\epsilon \zeta}{\eta} \mathbf{E}^{\prime}=-\beta \frac{\epsilon \zeta k_{B} T}{\eta q} \nabla \log \left(c_{0}\right)
$$

The diffusio-osmotic velocity for charged species becomes:

$$
\mathbf{V}_{D O}=-D_{D O} \nabla \log c_{0}
$$

with

$$
D_{D O}=-\beta \frac{\epsilon \zeta}{\eta} \frac{k_{B} T}{q}+\frac{\epsilon}{2 \pi \eta}\left(\frac{k_{B} T}{q}\right)^{2} \log \left(1-\zeta^{2}\right)
$$

where the first term is the self-induced electrophoretic contribution and the second term is the complete expression for chemio-osmosis [60]. In practice, the chemio-osmotic contribution is often negligible 10 As in electrophoresis, a

\footnotetext{
${ }^{9}$ For the sake of simplicity, we assume that anions and cations have the same absolute charge.

${ }^{10}$ Comparing $\mathrm{LiCl}$ and $\mathrm{KCl}$ electrolytes, in controlled microfluidic gradients, the diffusiophoretic mobility of latex or silica particles in $\mathrm{KCl}$ concentration gradients, $\beta_{\mathrm{KCl}} \sim 0$, was reported only $\sim 10 \%$ of the diffusio-phoretic mobility in $\mathrm{LiCl}$ gradients, for which $\beta$ is larger [72, 73,74 .
} 
colloid migrates diffusiophoretically in an solute gradient, with $\mathbf{V}_{D P}=-\mathbf{V}_{D O}$.

Important features of phoresis for the design of active colloids summarize:

- The flow relies on interaction of a solute species with the solid substrate, e.g. electrostatics for the electrophoresis.

- The interaction range is nanometric. The osmotic velocity appears, macroscopically, as an apparent slip velocity.

- The velocity is independent of geometry of homogenous particles of size $\mathrm{R}$, provided $R \gg \lambda$. Phoresis is robust to downsizing.

\section{Active Patchy Particles}

\subsection{Self-propelled Nanorods}

A natural avenue for self-propelled particles is the design of particles with fore-aft asymmetry and using phoretic phenomena. A pioneering realization is the making of gold-platinum bimetallic nanorods immersed in a solution of hydrogen peroxide $\left.\mathrm{H}_{2} \mathrm{O}_{2}, 75,76,77,78\right]$. The nanorods are typically $2 \mu \mathrm{m}$ in length and 200nm in diameter [Fig.1D] and propel with a velocity $V \sim 15 \mu \mathrm{m} / \mathrm{s}$. They exhibit a persistent random walk, though more subtle dynamics due to the curvature of the nanorods have been reported [79]. The system constitutes a short-circuited battery, with a platinum anode and a gold cathode. The addition of an insulating section between the electrodes suppresses the propulsion. The electrochemical decomposition of $\mathrm{H}_{2} \mathrm{O}_{2}$ results in a gradient in proton concentration, associated to a self-induced electric field pointing from $\mathrm{Pt}$ to $\mathrm{Au}$. It sets into motion the positive double layer surrounding the negatively charged nanorod, exhibiting a self-electrophoretic propulsion with the platinum heading [Fig.1E]. Details on reaction-induced charge auto-electrophoresis can be found in [80, 81. The non-equilibrium electric field is not screened by the static double layer and results in long-range interactions between pairs of nanorods 82 or tripartite Au-Pt-Au rods which assemble into stable rotors [83. It also accounts for the surprising increase of the propulsion velocity of nanorods in confining 
channels 84.

Numerous modifications have been performed on the original gold-platinum design. For example, the addition of magnetic segments allow to steer them externally [76, 85, 86. Different combinations of metals (Pt, Pd, Ni, $\mathrm{Au}, \mathrm{Ru}$, and $\mathrm{Rh}$ ) modify the difference of mixed potential between the electrodes and result in changes in speed and direction of propulsion [87. The addition of Carbon NanoTubes (CNT) within the platinum segment enhances the electrontransfer and leads to speeds up $\sim 100 \mu \mathrm{m} / \mathrm{s}$. Similarly, the addition of hydrazine to the solution enhances the decomposition of hydrogen peroxide and lead to speed $\sim 200 \mu \mathrm{m} / \mathrm{s}$ [88. A contrario, the addition of salt reduces the speed of the nanorods (unless it is silver salt, which deposits on the gold segment and increases the mixed potential difference between the electrodes) [89]. The phenomenon can be qualitatively understood since the driving stress (the motion of the double layer due to the auto-induced electric field) scales as the Debye length $\lambda_{D}, \sigma_{D} \propto \lambda_{D}$, and the viscous dissipation as the shear rate $\sigma_{\eta} \propto \frac{V}{\lambda_{D}}$ leading to a propulsion velocity $V \propto \lambda_{D}^{2} \propto 1 / \rho_{0}$, decaying with the salt concentration $\rho_{0}$.

\subsection{Autophoretic swimmers}

An alternate design is the Janus colloid, where only one side of the particle is active 90, 91. Such particles are typically synthesized by sputtering of a material $(\mathrm{Au}, \mathrm{Pt}, \mathrm{C} \ldots)$ on a monolayer of homogeneous spheres [Fig. 1F,G].

\subsubsection{Diffusio-phoretic swimmers}

Platinum-dielectric Janus, a model system?

Diffusio-phoretic swimmmers exhibit catalytic patches on dielectric particles 90. 91. They self-propel, in the auto-induced concentration gradient, with velocity $V_{p} \propto \frac{\alpha \mu}{D}$, which depends on (i) $\alpha$, the reaction rate of the reaction on the catalytic site, (ii) $\mu$, the diffusio-phoretic mobility of the particle in the concentration gradient of the considered specie [See Eq. 3 and (iii) $D$, the diffusion coefficient of the chemical in the medium 90, 91. The prefactor depends on the geometry of the patches on the particle. (i) and (ii) are surface properties of 
the particle, while (iii) depends on the medium. In this simple phoretic picture, the propulsion velocity is independent of the particle size. However, the concentration gradient is set by the particle itself, which can lead to size dependence of the velocity. The velocity of Pt-PS janus particles exhibits size-dependence, ${ }_{185} V_{p} \propto 1 / R$, for radii in the range $[250 \mathrm{~nm}-5 \mu \mathrm{m}]$. This can be simply understood in the diffusion limited regime, where the concentration of fuel at the active patch is 0 and the bulk concentration $C_{0}$, on the inert side, leading to $V_{p}=\mu \nabla C \propto \mu \frac{C_{0}}{R}$ [92]. The relationship between the geometry and the speed for self-phoretic Janus particles was recently discussed in [93].

Diffusio-phoretic swimmers were first obtained by sputtering a nanometric platinum cap on polystyrene spheres [Fig.1F] and immersed in hydrogen peroxide (typically $0.1-10 \%$ ) [52]. Platinum is a catalyst for the decomposition of hydrogen peroxide, $2 \mathrm{H}_{2} \mathrm{O}_{2}=2 \mathrm{H}_{2} \mathrm{O}+\mathrm{O}_{2}$ and the particle creates a concentration gradient. The velocity follows a Michaelin-Menten behavior with the concentration of fuel, suggesting a two-step process where hydrogen peroxide first forms a complex on platinum before breaking down in water and oxygen [52. The particles propel at speeds up to $\sim 10-15 \mu \mathrm{m} / \mathrm{s}$.

This system was initially considered a model system for the study of active colloids. The particles are conveniently synthesized: platinum can be sputtered on a wide range of commercially available dielectric, e.g. polystyrene or silica colloids. The yield is often limited by the intrinsic $2 \mathrm{D}$ nature of the process. Novel approaches have since been proposed to scale up the synthesis 94. The chemical decomposition of hydrogen peroxide, $2 \mathrm{H}_{2} \mathrm{O}_{2}=2 \mathrm{H}_{2} \mathrm{O}+\mathrm{O}_{2}$, only involves neutral species, which presumably simplifies the dependence of the selfpropulsion on surface chemistry, such as the $\zeta$ potential of the colloid or the $\mathrm{pH}$ of the solution. Along this line, neutral diffusio-phoresis was shown to provide an explanation for the observed reorientation of propelling Pt/dielectric Janus, with their hemisphere perpendicular to the substrate surface [95, 96. However, subsequent results hint that charged species are involved and that neutral diffusio-phoresis alone can not account for. The swimming velocity is 
strongly reduced by the presence of salt (and ionic impurities) and the direction of swimming is reversed in correlation with a change or $\zeta$ potential of the particle 97, 98,). Similarly the addition of cationic surfactant CTAB allows the direction of self-propulsion to be changed. The strong effects of pH-neutral salts would suggest ionic self-diffusiophoresis as an alternative mechanism of propulsion of the Pt-dielectric Janus particles but would require unrealistic potential for the colloids, $\zeta \sim 30 \mathrm{~V}$, to account for observed velocities ${ }^{11}$ 96. However, a mechanism of self-electrophoresis, akin to the self-propulsion of the nanorods, is compatible with the experimental data. The phenomenon is argued to emerge from different spatial areas involving oxidations and reductions on the cap and akin to the short-circuited battery [96] or the thickness-dependent reaction rate of the decomposition, larger at the pole than at the equator, leading to a flux of protons [99]. Further work is required to better understand this seemingly simple system, since even the direction of propulsion was reported to depend on the thickness of the platinum cap [96]!

\section{Light-activated Diffusiophoretic Swimmers}

Light-activated swimmers provide a convenient class of systems, where the propulsion is switched on and off externally. Light can be modulated spatially and temporally or with different wavelengths [100, offering routes towards complexity without experimental complications, and providing a fast switch to return to equilibrium, thanks to the fast diffusion of molecular species $(<1 \mathrm{~ms}$ for $1 \mu \mathrm{m})$.

Catalytic swimmers, where the platinum is replaced by a photocatalytic material activated by light, were subsequently proposed. Hematite, $\left(\mathrm{Fe}_{2} \mathrm{O}_{3}\right)$ [101], partially embedded in a dielectric material and immersed in hydrogen peroxide $(\mathrm{pH}=6.5)$ self-propels in $3 \mathrm{D}$ under blue illumination. So does titania $\left(\mathrm{TiO}_{2}\right.$, anatase phase), under illumination by UVA (390nm) [100]. We infer that semi-

\footnotetext{
${ }^{11}$ Indeed, diffusiophoretic velocities measured in microfluidics experiments were also significantly lower $V \sim 1-2 \mu \mathrm{m} / \mathrm{s}$ [72, 73, 74]
} 
conductors, illuminated above their bandgap, generate electron-holes pairs, and act as photocatalyst for the decomposition of $\mathrm{H}_{2} \mathrm{O}_{2}$. Alternatively, Janus titania particles exposed to UV, use water splitting to propel in an bio-compatible fuel [37.

An elegant example of light-activated propelled particles is provided by Janus silica-gold particles immersed in a critical binary mixture of water and -2,6lutidine. Under illumination, the gold cap heats up above the critical temperature and induces the demixing of the binary mixture. The particle subsequently propels in the established gradient and exhibits a persistent random walk with the velocity set by the local light intensity [54. Interestingly, the direction of propulsion is reversed by hydrophobically-functionalizing the gold cap, stressing the role of controlled patches to harness propulsion.

\subsubsection{Thermophoretic Swimmers}

Thermophoretic swimmers generate a thermal gradient resulting in thermophoretic propulsion [102]. For example, the gold cap of Janus gold-dielectric spheres heats up under under irradiation $(40 \mathrm{~mW}, 1064 \mathrm{~nm})$, setting a temperature gradient of $\sim 2 \mathrm{~K}$, leading to velocities of $10 \mu \mathrm{m} / \mathrm{s}$ [103. Similarly, thermophoretic propulsion was reported for silica particles partially covered with a magnetic material and exposed to an AC field. The hysteretic response of the magnetic material heats up of the cap, leading to self-propulsion in water [104].

\section{Other types of Swimmers}

Patchy swimmers

Silica spheres coated with metals and covered with a thin $\mathrm{SiO}_{2}$ protective layer (so that their surface properties are uniform) are immersed in deionized water and sedimented near a bottom substrate. Subject to an A.C. electric field perpendicular to the bottom substrate, the particles self-propel, isotropically, along the plane. The propulsion is due to the different polarizability of the two hemispheres exhibiting an asymmetric Induced Charge ElectroPhoresis (ICEP) 
leading to the propulsion of the particle with the dielectric heading [105, 106]. The propulsion of metallodielectric particles by ICEP was previously reported in 107. but the nifty use of an electric field perpendicular to the substrate plane made possible to obtain Janus swimmers with isotropic propulsion, though intrinsically planar. The system exhibits different collective behaviors tuning the interaction between particles thanks to the control of the amplitude and frequency of the A.C. electric field 108.

Catalytic locomotion of $\mathrm{Au} / \mathrm{Ru}$ core-shell nanowires was recently reported in the presence of hydrogen peroxide. Unlike conventional bimetallic nanowires, experimental observations pointed toward a propulsion from combined selfdiffusiophoresis and electroomosis in the oxygen gradient [109].

\section{Isotropic Swimmers}

In this section, we briefly review examples of isotropic particles, which nonetheless propel. The propulsion, a priori counter-intuitive, relies on a spontaneous symmetry-breaking mechanism.

The first example is provided by insulating spheres immersed in a conducting fluid. When an electric field $\mathbf{E}$ is applied above a critical amplitude, the particles start rotating at constant speed around a random direction transverse to E, this is the Quincke effect [110, 111]. Sitting near the bottom substrate, the particles start rolling at velocity $\sim \mathrm{mm} / \mathrm{s}[6]$.

Spontaneous autophoretic motion of isotropic particles has also been proposed theoretically. In a nutshell, a stagnant particle consuming fuel generates an isotropic concentration cloud. A random fluctuation moves the particle to one side, steepening the gradient on this side, possibly providing a route for spontaneous symmetry breaking. It was shown that this nonlinear interplay between surface osmotic flows (velocity U) and solute advection (diffusion D) can produce spontaneous and self-sustained motion of isotropic particles of radii $R$. It needs to distort the chemical cloud to amplify the fluctuation, i.e. $P e=\frac{U R}{D}>3$

295 [12. This condition is difficult to realize experimentally with colloidal particles $P e \sim \frac{30 \mu \mathrm{m} / \mathrm{s} \times 3 \mu \mathrm{m}}{10^{3} \mu \mathrm{m}^{2} / \mathrm{s}} \sim 0.1$ but can be achieved with larger droplets [113] and sug- 
gests an interesting pathway to motility. The vicinity of an impermeable wall can, alternatively, break the symmetry and induce propulsion of active isotropic colloids [114] or guide self-propelled Janus particles [115, 95].

\section{Remarks and Perspectives}

\section{Around diffusiophoresis, perspective and open questions}

This section stresses important features and questions raised by diffusiophoretic mechanisms, with possible impact and ramifications in natural and artificial situations. Interesting phenomena emerge when the interaction length of diffusiophoresis (or Debye length if the interaction is electrostatic), compares with the typical size of the system: the conductivity in a pore is larger than bulk conductivity when the Debye length becomes larger than the pore radius [116]. Similarly, the diffusiophoretic mobility becomes size dependence when the radius of a colloid compares with the interaction range [117.

Harnessing the subtlety of diffusiophoresis allows to sort particles by size in dead-end channels using solute gradients or super-imposition of salt gradients, at fixed total ionic strength [117, 118] or design long-range, surface-specific interactions in suspensions [119] or exclusion zone near certain walls [120].

315 The diffusiophoretic velocity is proportional to the gradient of the logarithm of ions, $\mathbf{V}_{D P}=D_{D P} \nabla \log c_{0}$. This can lead to the focusing of colloidal suspensions colloids [117, 72, 121 and raises the question of the sensitivity of diffusiophoretic migration for vanishingly small ionic concentrations, in particular in the ultra dilute-limit, where the inter-ions distance compares with the colloid radius. The potential relevance of diffusiophoresis (and phoretic transport) within the context of complex fluids is an open question: it has been predicted that diffusiophoresis of a rigid sphere in a Carreau fluid differs significantly from the Newtonian case [122] and reported that colloidal particles oscillate at the constriction of a microchannel under electrophoresis in a viscoelastic fluid [123]. 

often injected into reservoir to increase oil recovery [124. The possibility to replace salty water in this process by dissolved and dissociating gas, such as carbon dioxide, has not been explored. Diffusiophoresis offers numerous opportunities owing to its subtlety and provides uncharted (non-equilibrium) territories to explore, from oil-extraction to the origin of life [125].

\section{Comments on Active Patchy Swimmers}

In this last section, we discuss the challenges and opportunities offered by phoretic patchy particles. First, it can be difficult to properly describe the propulsion mechanism of the active particles. They often sit near a surface, a possible source of osmotic flow, which can contribute to the propulsion. Similarly, tracer particles are often phoretically active, which needs to be accounted to extract the hydrodynamic flow field [126]. Phenomena altering the surface of the particles can affect phoresis: surfactants, adsorbing polymers, and surface chemistry in general. Experiments need to be performed with cleaned solutions and chambers and in buffer solutions in order to control the surface chemistry. Roughness (comparable to the interaction length) or hydrodynamic slip can also have important and non trivial effects on the phoretic effect [127, 128, 71]. In spite of the conceptual simplicity, interfacial phenomena often intertwine different phenomena, e.g. (thermophoresis + diffusiophoresis), or superimpose in a non-linear fashion, as recently pointed out [118. Hydrogen peroxide as a fuel is a practical concern for biological application: ${ }^{12}$, which motivated the development of self-propelled particles in bio-compatible fuels and ultrasounds or bubble propelled swimmers [25, 26]. The dependability of phoretic swimmers on low ionic strength is an intrinsic weakness of the mechanims and limits their relevance in the bio-engineering context.

This complexity, on the other hand, offers opportunities for a high level of

\footnotetext{
${ }^{12}$ FDA recommends its use for sterilization at concentration $>2 \%$
} 
control and novel functionalities. The importance of surface chemistry allows to tailor the phoretic response of colloids thanks to chemically controlled patches 129, 130, 131. Particles with non-uniform surface properties or shapes [132, 133, 134 can be used to design spinners or reconfigurable structures. Semidilute suspensions could potentially be used as active depletants to direct selfassembly. This would however require non-interacting particles, in contradiction with the long-range disturbance of the concentration field surrounding phoretic swimmers and predicted to induce dynamic clusters [135] and wave patterns [136. Additionally, unless nanometric swimmers are used, the small number of active particles is likely to lead to intermittent attraction, as previously observed in bacterial suspensions [137, and qualitatively different from an equilibrium counterpart of depletion with "hot depletants". The opportunities offered are broad and largely untapped, providing a formidable playground where colloidal scientists, engineers and physicists can meet.

\section{Conclusion}

In this paper, we showed how phoretic transport is harnessed to design active patchy particles. The sensitivity of interfacial transport to surface properties $(\zeta$ potential, roughness, $\mathrm{pH} . .$.$) poses experimental challenges but also opens$ up opportunities for the colloidal scientist, which successfully control them. Numerous strategies have already been proposed to design propelled particles in a variety of fuel, or actuation mechanism. Activity beyond translational propulsion using patches has been, in large, unexplored. Their use to direct

375 colloidal self-assembly, such as machines to weave and bread on the microscale as recently proposed by Goodrich \& Brenner [138, is an uncharted territory.

\section{Acknowledgements}

JP thanks L. Bocquet for fruitful discussions, N. Agre for critical reading and the referee for her/his deep and thoughtful review. This work was supported 
1554724 .

\section{References}

[1] Marchetti MC, Joanny JF, Ramaswamy S, Liverpool TB, Prost J, Rao M, et al. Hydrodynamics of soft active matter. Reviews of Modern Physics

[7] Bricard A, Caussin JB, Das D, Savoie C, Chikkadi V, Shitara K, et al. Emergent vortices in populations of colloidal rollers. Nature Communications $2015 ; 6: 1-8$.

[8] Bechinger C, Di Leonardo R, Löwen H, Reichhardt C, Volpe G, Volpe G. Active Particles in Complex and Crowded Environments. Reviews of Modern Physics 2016;88(4):045006-50. 

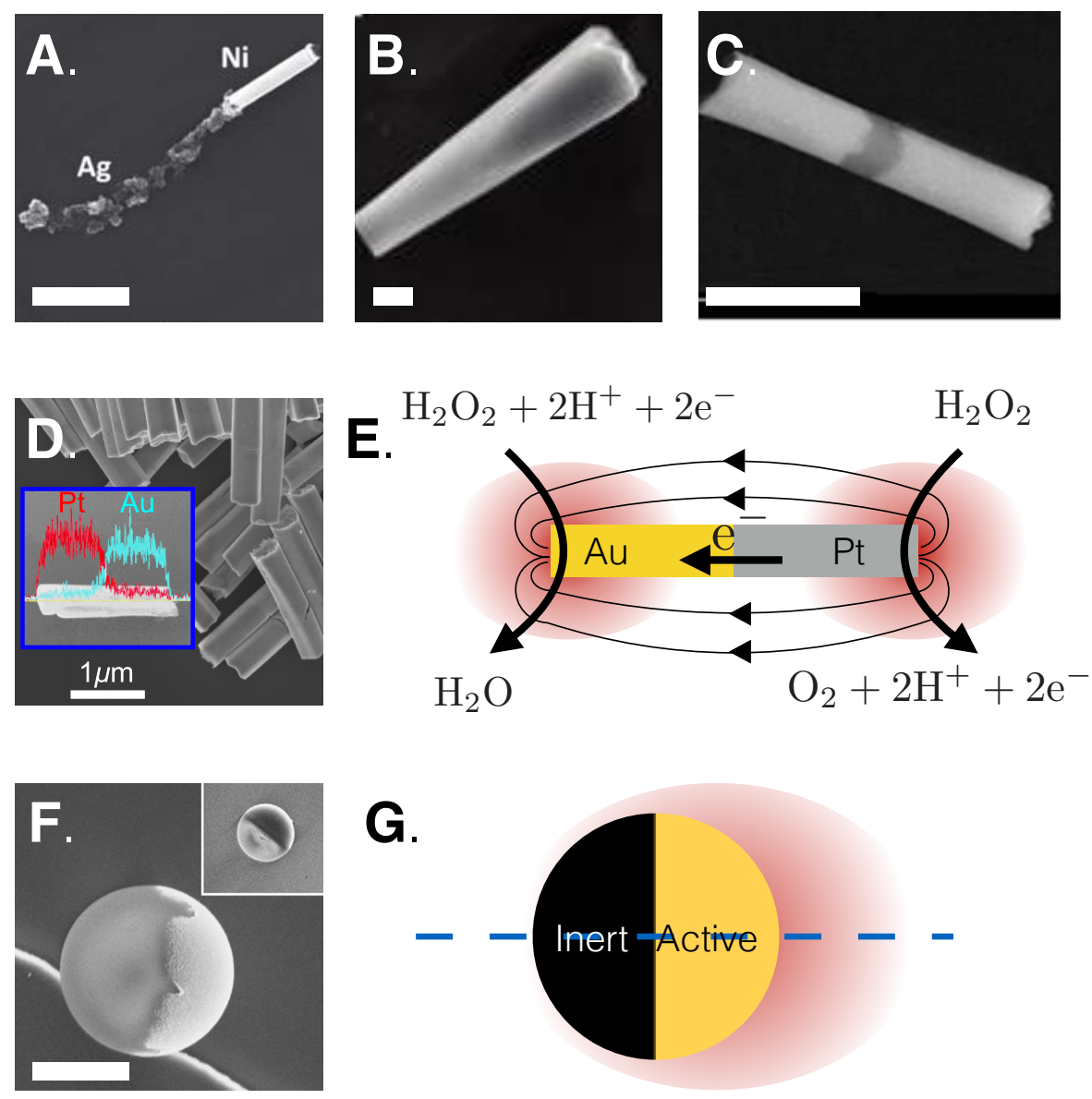

Figure 1: Overview of colloidal swimmers. (A) Self-propelled nanowire actuated by a magnetic field (reprint from [31]). (B) Bubble propelled microtube (reprint from [139]). (C) Ultrasonically propelled metal microrods micromotors (reprint from 140]). (D) Self-propelled $\mathrm{Pt} / \mathrm{Au}$ nanorods (reprint from [79]). (E) Mechanism of propulsion of Pt/Au nanorods in hydrogen peroxide. The scheme shows the electrochemical reactions, the approximate charge density (red cloud) and electric fields line. The rod self-propels to the right on the scheme (reprint from [0] ). (F) Scanning electron Microscopy image of a Platinum-dielectric Janus swimmer. Top corner, optical microscopy image, the light part is the Pt cap (reprint from [95]) ( G) Janus particle, one side is active, the other is inert. The particle sets up a gradient (red cloud) leading to phoretic propulsion along the poles (blue line). The direction of the propulsion depends on the details of the microscopic mechanism. Scale bars are $1 \mu \mathrm{m}$. 

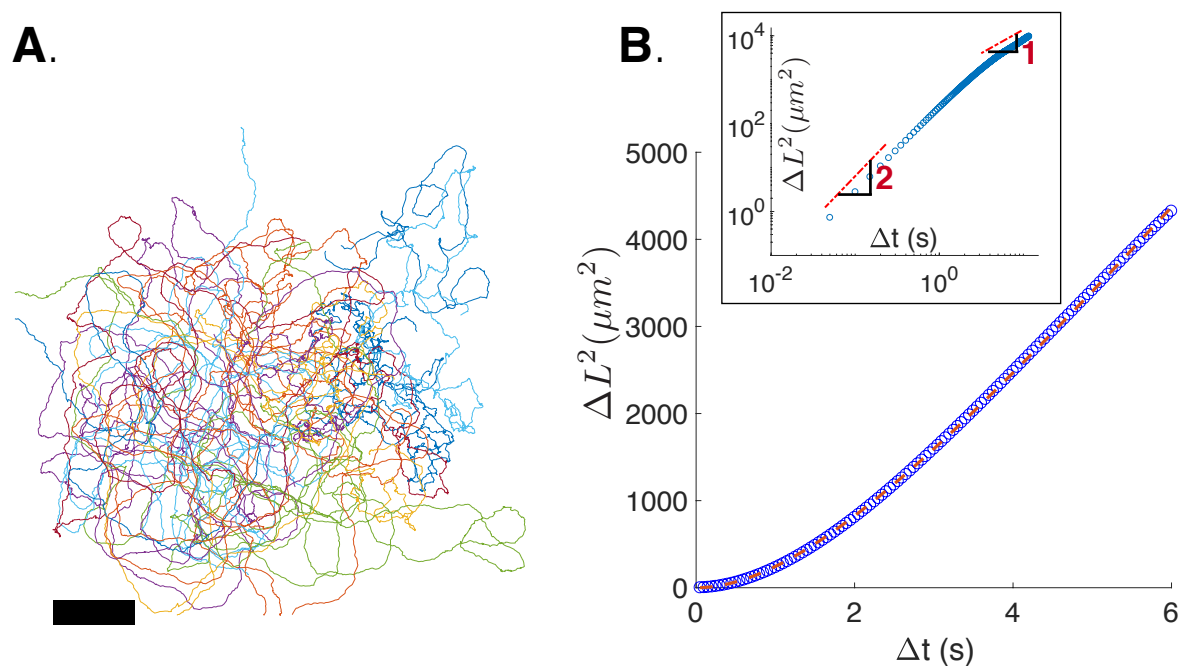

Figure 2: Individual dynamics of a swimmer. (A) Trajectories of photoactivated swimmers. The particle exhibits a persistent random walk. Scale bar is $20 \mu \mathrm{m}$. (B) Mean Square displacement $\Delta L^{2}$ obtained from the ensemble average of 24 individual trajectories (blue symbols) fit by [Eq1] (red dashed line), giving $V_{p}=17 \mu \mathrm{m} / \mathrm{s}$ and $\tau_{p}=3.5 \mathrm{~s}$. The equilibrium diffusion coefficient $D_{0}=0.030 \mu \mathrm{m}^{2} / \mathrm{s}$ is measured independently, and averaged over 249 particles. (Binset). Log-log plot of the MSD $\Delta L(\Delta t)$ showing the ballistic regime (slope 2) at short times, and diffusive regime (slope 1) for $t \gg \tau_{r}$. 


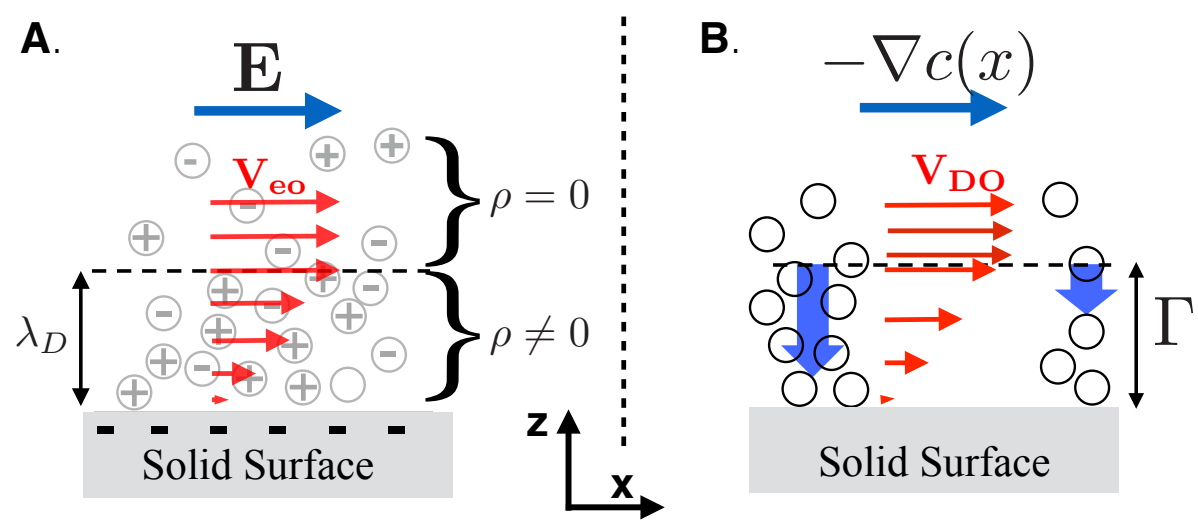

Figure 3: Interfacial Transport (A) Electro-osmosis. A solid surface immersed in an aqueous electrolyte is negatively charged.Cations are attracted to the surface, anions are repelled. They form a screening double layer of thickness $\lambda_{D}$ (see main text). An electric field $\mathbf{E}$ exerts a force on the non neutral double layer and sets the layer in motion. The outside layer is neutral and experiences no net force. The driving force is balanced by viscous dissipation leading to a constant flow velocity $\mathbf{V}_{e o}$ outside the Debye layer. (B) Diffusio-osmosis, flow under a concentration gradient $\nabla c(x)$. The solute is attracted to the substrate, the concentration gradient $\nabla c(x)$ induces an unbalanced osmotic pressure (blue arrows) within the interaction layer $\Gamma$ (notations defined in the main text), which drives the fluid in motion. This driving force is balanced by viscous dissipation and leads to a constant flow velocity $\mathbf{V}_{D O}$. 
[9] Takatori SC, Brady JF. Forces, stresses and the (thermo?) dynamics of active matter. Current Opinion in Colloid \& Interface Science 2016;21:2433.

[10] Cates ME, Tailleur J. When are active Brownian particles and run-andtumble particles equivalent? Consequences for motility-induced phase separation. EPL (Europhysics Letters) 2013;101(2):20010.

[11] Tailleur J, Cates ME. Sedimentation, trapping, and rectification of dilute bacteria. Europhysics Letters 2009;86(6):60002 (6pp).

[12] Marchetti MC, Fily Y, Henkes S, Patch A, Yllanes D. Minimal model of active colloids highlights the role of mechanical interactions in controlling the emergent behavior of active matter. Current Opinion in Colloid \& Interface Science 2016;21(C):34-43.

[13] Fily Y, Henkes S, Marchetti MC. Freezing and phase separation of selfpropelled disks. Soft Matter 2014;10(13):2132.

[14] Bialké J, Speck T, Löwen H. Crystallization in a Dense Suspension of Self-Propelled Particles. Physical Review Letters 2012;108(16):168301.

[15] Wittkowski R, Tiribocchi A, Stenhammar J. Scalar $\varphi^{4}$ field theory for active-particle phase separation. Nature communications 2014;.

[16] Solon AP, Fily Y, Baskaran A, Cates ME, Kafri Y, Kardar M, et al. Pressure is not a state function for generic active fluids. Nature Physics 2015;11:673-8.

[17] Solon AP, Stenhammar J, Wittkowski R, Kardar M, Kafri Y, Cates ME, et al. Pressure and Phase Equilibria in Interacting Active Brownian Spheres. Physical Review Letters 2015;114(19):198301.

[18] Takatori SC, Yan W, Brady JF. Swim Pressure: Stress Generation in Active Matter. Physical Review Letters 2014;113(2):028103-5. 
[19] Ray D, Reichhardt C, Reichhardt CJO. Casimir effect in active matter systems. Physical Review E 2014;90(1):013019-5.

[20] Bialké J, Siebert JT, Löwen H, Speck T. Negative Interfacial Tension in Phase-Separated Active Brownian Particles. Physical Review Letters 2015;115(9):098301.

[21] Ginot F, Theurkauff I, Levis D, Ybert C, Bocquet L, Berthier L, et al. Nonequilibrium Equation of State in Suspensions of Active Colloids. Physical review X 2015;5(1):011004-8.

[22] Takatori SC, De Dier R, Vermant J, Brady JF. Acoustic trapping of active matter. Nature Communications 2016;7:1-7.

[23] Wang W, Duan W, Ahmed S, Mallouk TE, Sen A. Small power: Autonomous nano- and micromotors propelled by self-generated gradients. Nano Today 2013;8(5):531-54.

[24] Dey KK, Wong F, Altemose A, Sen A. Current Opinion in Colloid \& Interface Science. Current Opinion in Colloid \& Interface Science 2016;21:4-13.

[25] Parmar J, Ma X, Katuri J, Simmchen J, Stanton MM, Trichet-Paredes C, et al. Nano and micro architectures for self- propelled motors. Science and Technology of Advanced Materials 2015;16(1):1-9.

[26] Singh VV, Wang J. Nano/micromotors for security/defense applications. A review. Nanoscale 2015;7(46):19377-89.

[27] Dey KK, Wong F, Altemose A, Sen A. Catalytic Motors-Quo Vadimus. Current Opinion in Colloid \& Interface Science 2016;21(C):4-13.

[28] Purcell EM. Life at low Reynolds number. American Journal of Physics $1977 ; 45(1): 3-11$.

455 [29] Lauga E, Powers TR. The hydrodynamics of swimming microorganisms. Reports On Progress In Physics 2009;72(9):096601. 
[30] Dreyfus R, Baudry J, Roper M, Fermigier M, Stone H, Bibette J. Microscopic artificial swimmers. Nature communications 2005;437(7060):862-5.

[31] Pak OS, Gao W, Wang J, Lauga E. High-speed propulsion of flexible nanowire motors: Theory and experiments. Soft Matter 2011;7(18):816981.

[32] Tierno P, Gueell O, Sagues F, Golestanian R, Pagonabarraga I. Controlled propulsion in viscous fluids of magnetically actuated colloidal doublets. Physical Review E 2010;81(1):011402.

[33] Gao W, Feng X, Pei A, Kane CR, Tam R, Hennessy C, et al. Bioinspired Helical Microswimmers Based on Vascular Plants. Nano Letters 2014;14(1):305-10.

[34] Gao W, Kagan D, Pak OS, Clawson C, Campuzano S, Chuluun-Erdene E, et al. Cargo-Towing Fuel-Free Magnetic Nanoswimmers for Targeted Drug Delivery. Small 2011;8(3):460-7.

[35] Driscoll M, Delmotte B, Youssef M, Sacanna S, Donev A, Chaikin PM. Unstable fronts and motile structures formed by microrollers. Nature Physics 2017;13:375-9.

[36] Gibbs JG, Zhao YP. Autonomously motile catalytic nanomotors by bubble propulsion. Applied Physics Letters 2009;94(16):163104.

[37] Gao W, Pei A, Wang J. Water-Driven Micromotors. Acs Nano 2012;6(9):8432-8.

[38] Solovev AA, Xi W, Gracias DH, Harazim SM, Deneke C, Sanchez S, et al. Self-Propelled Nanotools. Acs Nano 2012;6(2):1751-6.

${ }_{480}$ [39] Sanchez S, Ananth AN, Fomin VM, Viehrig M, Schmidt OG. Superfast Motion of Catalytic Microjet Engines at Physiological Temperature. Journal of the American Chemical Society 2011;133(38):14860-3. 
[40] Kagan D, Laocharoensuk R, Zimmerman M, Clawson C, Balasubramanian S, Kang D, et al. Rapid Delivery of Drug Carriers Propelled and Navigated by Catalytic Nanoshuttles. Small 2010;6(23):2741-7.

[41] Soto F, Wagner GL, Garcia-Gradilla V, Gillespie KT, Lakshmipathy DR, Karshalev E, et al. Acoustically propelled nanoshells. Nanoscale 2016;8(41):17788-93.

[42] Xu T, Soto F, Gao W, Garcia-Gradilla V, Li J, Zhang X, et al. UltrasoundModulated Bubble Propulsion of Chemically Powered Microengines. Journal of the American Chemical Society 2014;136(24):8552-5.

[43] Garcia-Gradilla V, Orozco J, Sattayasamitsathit S, Soto F, Kuralay F, Pourazary A, et al. Functionalized Ultrasound-Propelled Magnetically Guided Nanomotors: Toward Practical Biomedical Applications. Acs Nano 2013;7(10):9232-40.

[44] Patteson AE, Gopinath A, Arratia PE. Active Colloids in complex fluids. Current Opinion in Colloid \& Interface Science 2016;21:86-96.

[45] Lauga E. The bearable gooeyness of swimming. Journal of Fluid Mechanics $2014 ; 762: 1-4$.

[46] Schamel D, Mark AG, Gibbs JG, Miksch C, Morozov KI, Leshansky AM, et al. Nanopropellers and Their Actuation in Complex Viscoelastic Media. Acs Nano 2014;8(9):8794-801.

[47] Gómez S, Godínez FA, Lauga E, Zenit R. Helical propulsion in shearthinning fluids. Journal of Fluid Mechanics 2016;812:283-12.

[48] Gagnon DA, Keim NC, Arratia PE. Undulatory swimming in shearthinning fluids: experiments with Caenorhabditis elegans. Journal of Fluid Mechanics 2014;758:622-11.

[49] Elfring GJ, Lauga E. Theory of locomotion through complex fluids. Complex Fluids in Biological Systems; Springer; 2014. 
[50] Qiu T, Lee TC, Mark AG, Morozov KI, Münster R. Swimming by reciprocal motion at low Reynolds number. Nature communications 2014;5(5119).

[51] Dasgupta M, Liu B, Fu HC, Berhanu M, Breuer KS, Powers TR, et al. Speed of a swimming sheet in Newtonian and viscoelastic fluids. Physical Review E 2013;87(1):013015-7.

[52] Howse JR, Jones RAL, Ryan AJ, Gough T, Vafabakhsh R, Golestanian R. Self-motile colloidal particles: From directed propulsion to random walk. Physical Review Letters 2007;99(4).

[53] Palacci J, Cottin-Bizonne C, Ybert C, Bocquet L. Sedimentation and Effective Temperature of Active Colloidal Suspensions. Physical Review Letters 2010;105(8):088304.

[54] Buttinoni I, Volpe G, Kümmel F, Volpe G, Bechinger C. Active Brownian motion tunable by light. Journal of Physics: Condensed Matter 2012;24(28):284129.

[55] Barrat JL, Pierre Hansen J. Basic concepts for simple and complex liquids. Cambridge University Press; 2003.

[56] Liu B, ker ABx. Measuring rotational diffusion of colloidal spheres with confocal microscopy. Soft Matter 2016;12:6033-7.

[57] Cerbino R, Trappe V. Differential dynamic microscopy: probing wave vector dependent dynamics with a microscope. Physical Review Letters 2008;100(18):188102.

[58] Wilson LG, Martinez VA, Schwarz-Linek J, Tailleur J. Differential dynamic microscopy of bacterial motility. Physical Review Letters 2011;106(18):101.

[59] Wittmeier A, Leeth Holterhoff A, Johnson J, Gibbs JG. Rotational Analysis of Spherical, Optically Anisotropic Janus Particles by Dynamic Microscopy. Langmuir 2015;31(38):10402-10. 
[60] Anderson JL. Colloid Transport by Interfacial Forces. Annual Review Of Fluid Mechanics 1989;21:61-99.

[61] Squires TM, Quake SR. Microfluidics: Fluid physics at the nanoliter scale. Reviews of Modern Physics 2005;77(3):977-1026.

[62] Persat A, Santiago JG. An Ohmic model for electrokinetic flows of binary asymmetric electrolytes. Current Opinion in Colloid \& Interface Science $2016 ; 24(\mathrm{C}): 52-63$.

[63] Yariv E. "Force-free" electrophoresis? Physics of Fluids 2006;18(3):031702-5.

[64] von Smoluchowski M. Zur kinetischen theorie der brownschen molekularbewegung und der suspensionen. Annalen der physik 1906;326(14):756-80.

[65] Morrison FA. Electrophoresis of a Particle of Arbitrary Shape. Journal Of Colloid And Interface Science 1970;34(2):210-4.

[66] Derjaguin BV, Sidorenkov GP, Zubashchenkov EA, Kiseleva EV. Kinetic phenomena in boundary films of liquids. Kolloidn Zh 1947;9:335-47.

[67] Prieve DC, Anderson JL, Ebel JP, Lowell ME. Motion of a Particle Generated by Chemical Gradients .2. Electrolytes. Journal of Fluid Mechanics 1984;148:247-69.

[68] Anderson JL, Lowell ME, Prieve DC. Motion of a Particle Generated by Chemical Gradients .1. Non-Electrolytes. Journal of Fluid Mechanics $1982 ; 117: 107-21$.

[69] Velegol D, Garg A, Guha R, Kar A, Kumar M. Origins of concentration gradients for diffusiophoresis. Soft Matter 2016;12:4686-703.

[70] Keh HJ. Diffusiophoresis of charged particles and diffusioosmosis of electrolyte solutions. Current Opinion in Colloid \& Interface Science $2016 ; 24: 13-22$. 
[71] Ajdari A, Bocquet L. Giant amplification of interfacially driven transport by hydrodynamic slip: Diffusio-osmosis and beyond. Physical Review Letters 2006;96(18):186102.

[72] Palacci J, Abecassis B, Cottin-Bizonne C, Ybert C, Bocquet L. Colloidal Motility and Pattern Formation under Rectified Diffusiophoresis. Physical Review Letters 2010;104(13).

[73] Abecassis B, Cottin-Bizonne C, Ybert C, Ajdari A, Bocquet L. Osmotic manipulation of particles for microfluidic applications. New Journal of Physics 2009;11(7):075022.

[74] Abecassis B, Cottin-Bizonne C, Ybert C, Ajdari A, Bocquet L. Boosting migration of large particles by solute contrasts. Nature Materials 2008;7:785-789.

575 [75] Paxton WF, Sundararajan S, Mallouk TE, Sen A. Chemical locomotion. Angewandte Chemie-International Edition In English 2006;45(33):5420-9.

[76] Kline T, Paxton W, Mallouk T, Sen A. Catalytic nanomotors: Remotecontrolled autonomous movement of striped metallic nanorods. Angewandte Chemie-International Edition In English 2005;44(5):744-6.

[77] Kline TR, Paxton WF, Wang Y, Velegol D, Mallouk TE, Sen A. Catalytic micropumps: Microscopic convective fluid flow and pattern formation. Journal of the American Chemical Society 2005;127(49):17150-1.

[78] Paxton W, Kistler K, Olmeda C, Sen A, St Angelo S, Cao Y, et al. Catalytic nanomotors: Autonomous movement of striped nanorods. Journal of the American Chemical Society 2004;126(41):13424-31.

[79] Takagi D, Braunschweig A, Zhang J, Shelley M. Dispersion of SelfPropelled Rods Undergoing Fluctuation-Driven Flips. Physical Review Letters 2013;110(3):038301. 
[80] Moran JL, Posner JD. Electrokinetic locomotion due to reaction-induced charge auto-electrophoresis. Journal of Fluid Mechanics 2011;680:31-66.

[81] Wang W, Chiang TY, Velegol D, Mallouk TE. Understanding the Efficiency of Autonomous Nano- and Microscale Motors. Journal of the American Chemical Society 2013;135(28):10557-65.

[82] Wang W, Duan W, Sen A, Mallouk TE. Catalytically powered dynamic assembly of rod-shaped nanomotors and passive tracer particles. Proceedings Of The National Academy Of Sciences Of The United States Of America 2013;110(44):17744-9.

[83] Wykes MSD, Palacci J, Adachi T, Ristroph L, Zhong X, Ward MD, et al. Dynamic self-assembly of microscale rotors and swimmers. Soft Matter 2016;12(20):4584-9.

[84] Liu C, Zhou C, Wang W, Zhang HP. Bimetallic Microswimmers Speed Up in Confining Channels. Physical Review Letters 2016;117(19):198001.

[85] Burdick J, Laocharoensuk R, Wheat PM, Posner JD, Wang J. Synthetic Nanomotors in Microchannel Networks: Directional Microchip Motion and Controlled Manipulation of Cargo. Journal of the American Chemical Society $2008 ; 130(26): 8164-5$.

[86] Sundararajan S, Sengupta S, Ibele ME, Sen A. Drop-Off of Colloidal Cargo Transported by Catalytic Pt-Au Nanomotors via Photochemical Stimuli. Small 2010;6(14):1479-82.

[87] Wang Y, Hernandez RM, Bartlett DJ, Bingham JM, Kline TR, Sen A, et al. Bipolar electrochemical mechanism for the propulsion of catalytic nanomotors in hydrogen peroxide solutions. Langmuir 2006;22(25):104516.

[88] Laocharoensuk R, Burdick J, Wang J. Carbon-Nanotube-Induced Acceleration of Catalytic Nanomotors. Acs Nano 2008;2(5):1069-75. 
[89] Kagan D, Calvo-Marzal P, Balasubramanian S, Sattayasamitsathit S, Manesh KM, Flechsig GU, et al. Chemical Sensing Based on Catalytic Nanomotors: Motion-Based Detection of Trace Silver. Journal of the American Chemical Society 2009;131(34):12082-3.

[90] Golestanian R, Liverpool TB, Ajdari A. Propulsion of a Molecular Machine by Asymmetric Distribution of Reaction Products. Physical Review Letters 2005;94:220801.

[91] Golestanian R, Liverpool TB, Ajdari A. Designing phoretic micro- and nano-swimmers. New Journal of Physics 2007;9(5):-126.

${ }_{625}$ [92] Ebbens S, Tu MH, Howse JR, Golestanian R. Size dependence of the propulsion velocity for catalytic Janus-sphere swimmers. Physical Review E 2012;85(2):020401-4.

[93] Nourhani A, Lammert PE. Geometrical Performance of Self-Phoretic Colloids and Microswimmers. Physical Review Letters 2016;116(17):1783025 .

[94] Lee VE, Sosa C, Liu R, Prud'homme RK, Priestley RD. Scalable Platform for Structured and Hybrid Soft Nanocolloids by Continuous Precipitation in a Confined Environment. Langmuir 2017;33(14):3444-9.

[95] Simmchen J, Katuri J, Uspal WE, Popescu MN, Tasinkevych M, Sanchez S. Topographical pathways guide chemical microswimmers. Nature Communications 2016;7.

[96] Brown A, Poon W. Ionic effects in self-propelled Pt-coated Janus swimmers. Soft Matter 2014;10(22):4016.

[97] Tse c, Lin YW, Jiang HR. Zeta potential dependent Self-electrophoresis 640 of Pt-coated Janus particles in hydrogen peroxide solutions . arxiv 2017;https://arxiv.org/abs/1702.03743. 
[98] Palacci J, Sacanna S, Vatchinsky A, Chaikin PM, Pine DJ. Photoactivated Colloidal Dockers for Cargo Transportation. Journal of the American Chemical Society 2013;135(43):15978-81.

[99] Ebbens S, Gregory DA, Dunderdale G, Howse JR, Ibrahim Y, Liverpool TB, et al. Electrokinetic effects in catalytic platinum-insulator Janus swimmers. EPL (Europhysics Letters) 2014;106(5):58003.

[100] Palacci J, Sacanna S, Kim SH, Yi GR, Pine DJ, Chaikin PM. Lightactivated self-propelled colloids. Philosophical Transactions of the Royal Society A: Mathematical, Physical and Engineering Sciences 2014;372:20130372.

[101] Sugimoto T, Sakata K, Muramatsu A. Formation Mechanism of Monodisperse Pseudocubic $\alpha$-Fe2O3 Particles from Condensed Ferric Hydroxide Gel. Journal Of Colloid And Interface Science 1993;159(2):372-82.

[102] Piazza R. Thermophoresis: moving particles with thermal gradients. Soft Matter 2008;4(9):1740-4.

[103] Jiang HR, Yoshinaga N, Sano M. Active Motion of a Janus Particle by Self-Thermophoresis in a Defocused Laser Beam. Physical Review Letters 2010;105(26):268302.

[104] Baraban L, Streubel R, Makarov D, Han L, Karnaushenko D, Schmidt OG, et al. Fuel-Free Locomotion of Janus Motors: Magnetically Induced Thermophoresis. Acs Nano 2013;7(2):1360-7.

[105] Bazant MZ, Squires TM. Induced-charge electrokinetic phenomena: Theory and microfluidic applications. Physical Review Letters 2004;92(6):066101.

[106] Bazant MZ, Squires TM. Induced-charge electrokinetic phenomena. Current Opinion in Colloid \& Interface Science 2010;15(3):203-13. 
[107] Gangwal S, Cayre OJ, Bazant MZ, Velev OD. Induced-charge electrophoresis of metallodielectric particles. Physical Review Letters 2008;100(5):058302.

[108] Yan J, Han M, Zhang J, Xu C, Luijten E, Granick S. Reconfiguring active particles by electrostatic imbalance. Nature Materials 2016;15(10):1095-9.

[109] Jang B, Wang W, Wiget S, Petruska AJ, Chen X, Hu C, et al. Catalytic Locomotion of Core-Shell Nanowire Motors. Acs Nano 2016;10(11):998391.

[110] Melcher JR, Taylor GI. Electrohydrodynamics: a review of the role of interfacial shear stresses. Annual Review Of Fluid Mechanics 1969;1:11146.

[111] Saville DA. Electrohydrodynamics: the Taylor-Melcher leaky dielectric model. Annual Review Of Fluid Mechanics 1997;29:27-64.

[112] Michelin S, Lauga E, Bartolo D. Spontaneous autophoretic motion of isotropic particles. Physics of Fluids 2013;25(6):061701.

[113] Maass CC, Krüger C, Herminghaus S, Bahr C. Swimming Droplets. Annual Review of Condensed Matter Physics 2016;7(1):171-93.

[114] Yariv E. Wall-induced self-diffusiophoresis of active isotropic colloids. Physical Review Fluids 2016;1(3):032101-8.

[115] Uspal WE, Popescu MN, Dietrich S, Tasinkevych M. Guiding Catalytically Active Particles with Chemically Patterned Surfaces. Physical Review Letters 2016;117(4):048002-5.

${ }_{690}$ [116] Ho C, Qiao R, Heng JB, Chatterjee A, Timp RJ, Aluru NR, et al. Electrolytic transport through a synthetic nanometer-diameter pore. Proceedings Of The National Academy Of Sciences Of The United States Of America 2005;102(30):10445-50. 
[117] Shin S, Um E, Sabass B, Ault JT, Rahimi M, Warren PB, et al. Sizedependent control of colloid transport via solute gradients in dead-end channels. Proceedings Of The National Academy Of Sciences Ofx The United States Of America 2016;113(2):257-61.

[118] Shi N, Nery-Azevedo R, Abdel-Fattah AI, Squires TM. Diffusiophoretic Focusing of Suspended Colloids. Physical Review Letters 2016;117(25).

[119] Banerjee A, Williams I, Azevedo RN, Helgeson ME, Squires TM. Solutoinertial phenomena: Designing long-range, long-lasting, surface-specific interactions in suspensions. Proceedings of the National Academy of Sciences of the USA 2016;113(31):8612-7.

[120] Musa S, Florea D, Wyss HM, Huyghe JM. Convection associated with exclusion zone formation in colloidal suspensions. Soft Matter 2016;12:112732.

[121] Palacci J, Cottin-Bizonne C, Ybert C, Bocquet L. Osmotic traps for colloids and macromolecules based on logarithmic sensing in salt taxis. Soft Matter 2012;8(4):980-94.

[122] Tseng S, Su CY, Hsu JP. Diffusiophoresis of a charged, rigid sphere in a Carreau fluid. Journal Of Colloid And Interface Science 2016;465(C):54-7.

[123] Lu X, Patel S, Zhang M, Woo Joo S, Qian S, Ogale A, et al. An unexpected particle oscillation for electrophoresis in viscoelastic fluids through a microchannel constriction. Biomicrofluidics 2014;8(2):021802-11.

[124] Lager A, Webb KJ, Collins IR, Richmond DM. LoSal Enhanced Oil Recovery: Evidence of Enhanced Oil Recovery at the Reservoir Scale. In: SPE Symposium on Improved Oil Recovery, 20-23 April, Tulsa, Oklahoma, USA. Society of Petroleum Engineers; 2008,.

[125] Möller FM, Kriegel F, Kieß M, Sojo V, Braun D. Steep pH Gradients and Directed Colloid Transport in a Microfluidic Alkaline Hydrothermal Pore. Angewandte Chemie 2017;129(9):2380-4. 
[126] Moyses H, Palacci J, Sacanna S, Grier DG. Trochoidal trajectories of self-propelled Janus particles in a diverging laser beam. Soft Matter 2016;12(30):6357-64.

[127] Messinger RJ, Squires TM. Suppression of Electro-Osmotic Flow by Surface Roughness. Physical Review Letters 2010;105(14):144503.

[128] Khair AS, Squires TM. The influence of hydrodynamic slip on the electrophoretic mobility of a spherical colloidal particle. Physics of Fluids 2009;21(4):042001.

[129] Youssef M, Hueckel T, Yi GR, Sacanna S. Shape-shifting colloids via stimulated dewetting. Nature Communications 2016;7:1-7.

[130] Zheng X, Liu M, He M, Pine DJ, Weck M. Shape-Shifting Patchy Particles. Angewandte Chemie-International Edition In English 2017;56(20):550711.

735 [131] Sacanna S, Korpics M, Rodriguez K, Colon-Melandez L, Kim SH, Pine DJ, et al. Shaping colloids for self-assembly. Nature Communications 2013;4:1688-6.

[132] Sacanna S, Pine DJ. Current Opinion in Colloid \& Interface Science. Current Opinion in Colloid \& Interface Science 2011;16(2):96-105.

[133] Sacanna S, Pine DJ, Yi GR. Engineering shape: the novel geometries of colloidal self-assembly. Soft Matter 2013;9(34):8096-11.

[134] Manoharan VN. Colloidal matter: Packing, geometry, and entropy. Science 2015;349(6251):942.

[135] Yan W, Brady JF. The behavior of active diffusiophoretic suspensions: An accelerated Laplacian dynamics study. Journal of Chemical Physics 2016;145(13):134902-19. 
[136] Liebchen B, Marenduzzo D, Cates ME. Phoretic Interactions Generically Induce Dynamic Clusters and Wave Patterns in Active Colloids. arxiv 2017;https://arxiv.org/abs/1703.03245.

[137] Angelani L, Maggi C, Bernardini ML, Rizzo A, Di Leonardo R. Effective Interactions between Colloidal Particles Suspended in a Bath of Swimming Cells. Physical Review Letters 2011;107(13):138302.

[138] Goodrich CP, Brenner MP. Using active colloids as machines to weave and braid on the micrometer scale. Proceedings Of The National Academy Of Sciences Of The United States Of America 2017;114(2):257-62.

[139] Gao W, Sattayasamitsathit S, Orozco J, Wang J. Highly Efficient Catalytic Microengines: Template Electrosynthesis of Polyaniline/Platinum Microtubes. Journal of the American Chemical Society 2011;133(31):11862-4.

760 [140] Wang W, Castro LA, Hoyos M, Mallouk TE. Autonomous Motion of Metallic Microrods Propelled by Ultrasound. Acs Nano 2012;6(7):612232 . 